\title{
ENERGY ANALYZER EXPERIMENTS FOR THE UNIVERSITY OF MARYLAND ELECTRON RING
}

\author{
A. Valfells ${ }^{\dagger}$, S. Bernal, Y.P. Cui, R. A. Kishek, P.G. O’Shea, B. Quinn, M. Reiser, M. Virgo, V. Yun \\ University of Maryland, College Park, MD 20742-3511, USA. \\ M. Nishiura, National Institute for Fusion Science, Oroshi-cho, Toki, GIFU, 509-5292, Japan
}

\begin{abstract}
The University of Maryland electron ring (UMER) experiment $[1,2]$, currently under construction, will be used to investigate the dynamics of space charge dominated low energy $(10 \mathrm{keV})$ electron beams. One point of interest is the energy distribution in the beam. We use an energy analyzer of cylindrical geometry to determine the longitudinal energy of the electron beam. The data can be resolved temporally, which gives an indication of the axial energy distribution in the beamlets. We also have the capability of scanning the beam in the transverse direction so that a lateral distribution of the longitudinal energy may be determined. We present results of energy spread measurements from the injector region of UMER under various conditions. Later efforts will include the effects of bending on the energy spread in the beam.
\end{abstract}

\section{EXPERIMENTAL SETUP AND METHOD}

In our experiment we use a thermionic triode gun with a variable accelerating voltage ranging from 0 to $10 \mathrm{kV}$. The beam current is typically around $100 \mathrm{~mA}$. The pulse length is roughly $100 \mathrm{~ns}$ and the repetition rate is $60 \mathrm{~Hz}$. A solenoid is used to focus the beam into a diagnostic chamber where the energy analyzer is located. The energy analyzer is cylindrical with a focusing electrode that is electrically connected to the retarding mesh [3](see Figure 1). A collector plate is located downstream of the retarding mesh, and all of this is within a grounded casing with a $2 \mathrm{~mm}$ diameter pinhole at the front for beam entry. There is a mesh at the pinhole to minimize defocusing at the aperature. The source of the bias voltage of the analyzer is a power supply seperate from that used to supply the accelerating potential. We use the LabView program to control the potential to the energy analyzer (with our present equipment we can vary the retarding potential in $1 \mathrm{~V}$ steps), and to record the current signal from the collector plate. The analyzer is fastened to an arm in such a manner that it may moved across the electron beam (typically of $1 \mathrm{~cm}$ diameter). This lets the user sample the longitudinal energy across the beam profile. We also have the ability to insert a phosphor screen into the plane of the energy analyzer so that we may obtain an image of the beam at that axial position.

The process of measurement is the following: For a given retarding potential 300 current pulses are sampled

\footnotetext{
* This work was supported by the DoE

$\dagger$ agustv@glue.umd.edu
}

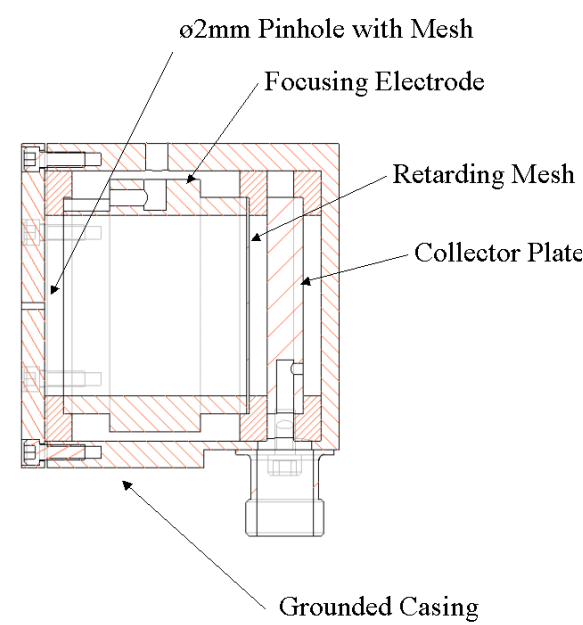

Figure 1: Cross Section of Energy Analyzer.

and averaged. Then the signal is filtered to eliminate the effects of ringing in the circuit. This process is repeated for a series of retarding potentials. Figure 2 shows the filtered signals for various values of the retarding potential for a beam of approximately $10 \mathrm{keV}$ energy.

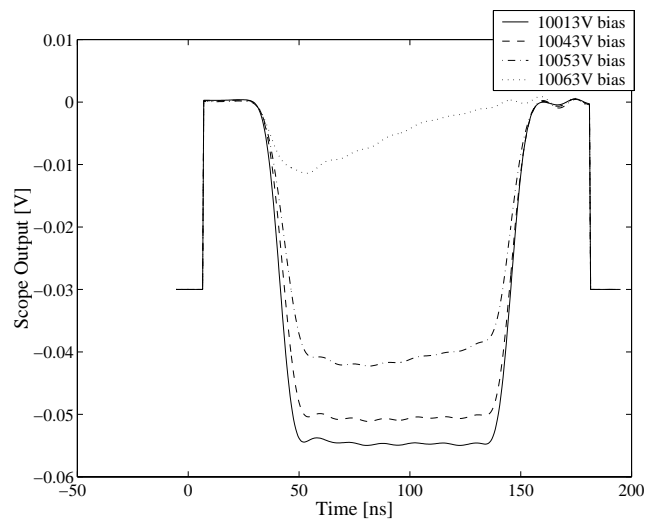

Figure 2: Scope Traces for Different Bias Voltages.

Because of the excellent pulse to pulse reproducibility it is possible to see how the collector signal varies with bias voltage at a given time in the pulse. Figure 3 shows such a curve taken mid-pulse. From this stage it is easy to construct a cumulative distribution function, and subsequently a probability density function for the beam energy. Figure 4 shows the energy distribution function corresponding to the curve of Figure 3. These measurements may then be 
taken for different settings of the pulse length, accelerating potential, and transverse position along the beam. All of the measurements presented in this paper were taken at the same axial position.

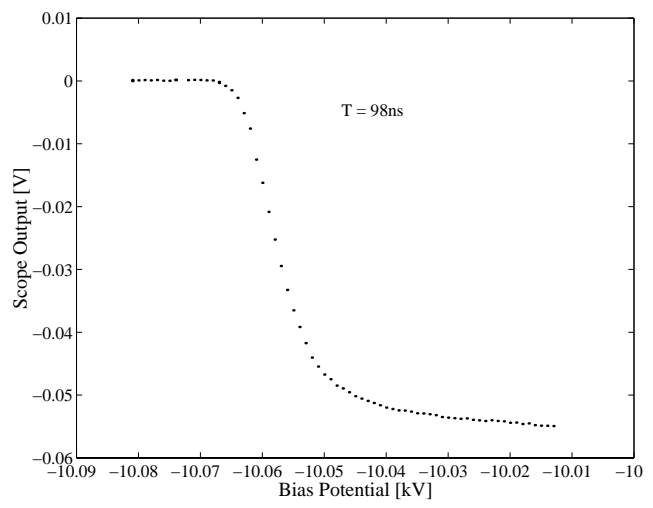

Figure 3: Pulse Amplitude as a Function of Bias Voltage; $\mathrm{T}=87 \mathrm{~ns}$.

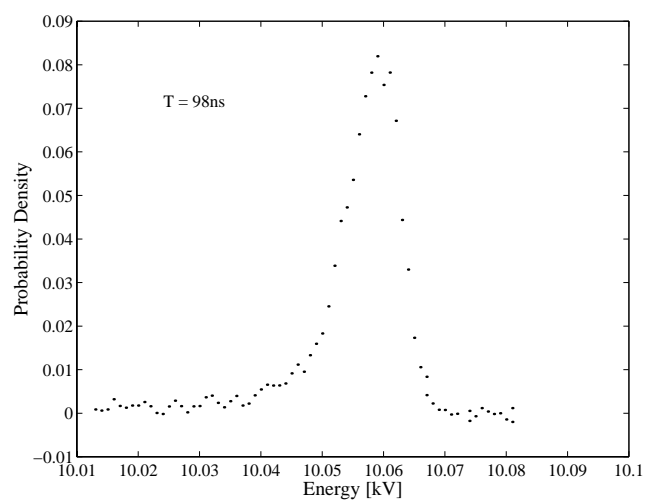

Figure 4: Measured Probability Density Function.

\section{RESULTS AND ANALYSIS}

We begin by examining the variation in the average energy and the energy spread for a $10 \mathrm{keV}$ beam with a current pulse shown in Figure 5. Note the arrows pointing to the head and the tail end of the beam. Figure 6 shows the corresponding energy distribution function for the head and tail of the beam.

To get a quantitative measure for the energy spread we define the energy spread as the square root of the variance of the measured probability density function. Figure 7 shows the calculated average energy for different times in the pulse shown in Figure 5. We note that there is a definite downwards trend in the variation of the mean energy. Figure 8 shows the measured energy spread for the aforementioned beam. Interestingly enough there is a clear decrease in the energy spread as one moves from the head of the beam to the tail of the beam. The wiggle is due to ringing in the circuit which is amplified during the differentiation process.

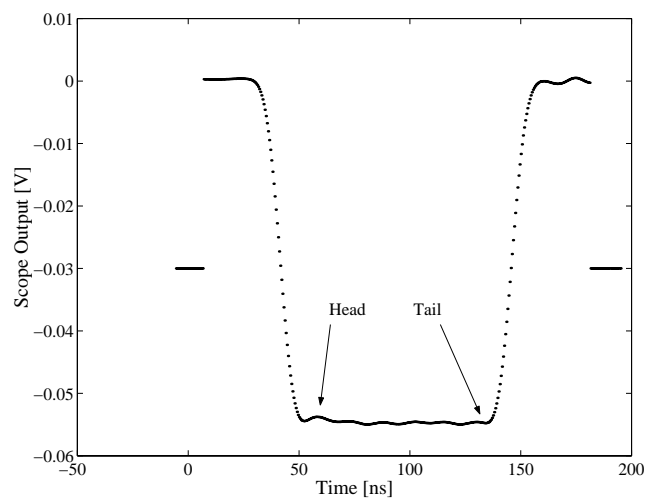

Figure 5: Current Pulse near Beam Center.

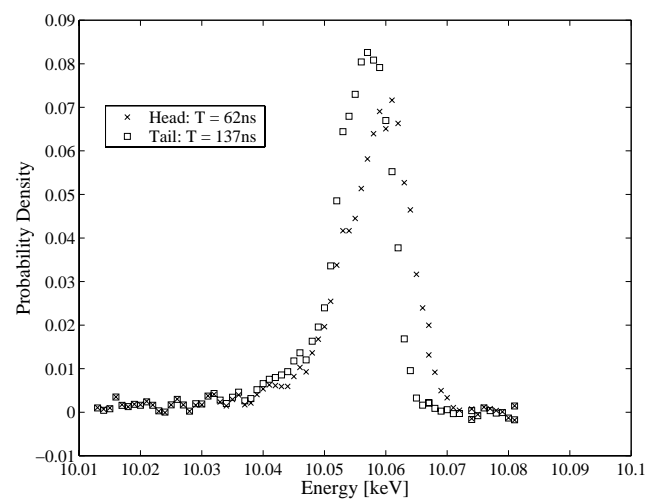

Figure 6: Energy Spread at Head and Tail.

The data described in the preceding paragraph was obtained when the aperature of the energy analyzer was located near the center of the beam. Let us now inspect the data obtained when the aperature is near the edge of the beam. First we notice a difference in the time-profile of the pulse (Figure 9), inasmuch as there is an apparent droop in the current pulse. The exact cause of this is unknown, though it may be tentatively attributed either to drooping in the gap voltage or it could also be due to the fact that the energy analyzer is in this case located at the position of

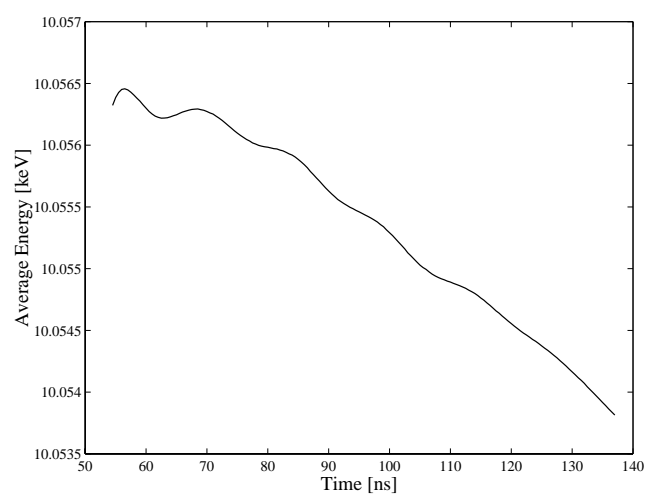

Figure 7: Temporal Variation of Average Energy. 


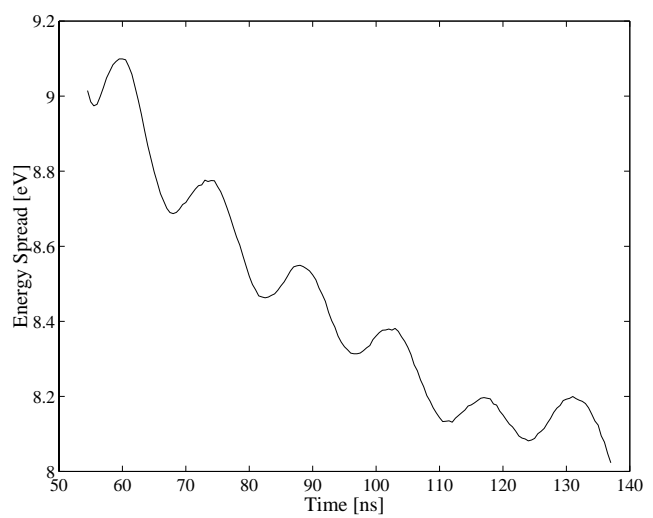

Figure 8: Temporal Variation of Energy Spread.

the crest of a density wave moving in the transverse direction [4] (we know from phosphor screen images that the analyzer is located at such a point in this case). We also notice a slight difference in the energy spread (Figure 10) and in the average energy (Figure 11). These differences in energy spread and average energy may be due to the fact that at the edge of the beam a greater number of electrons are entering the analyzer obliquely, which may manifest itself as an increased apparent spread and smaller average energy. We hope that an improved design with the possibility of keeping the focusing electrode at a slightly different potential than the retarding mesh will let us minimize this possible error.

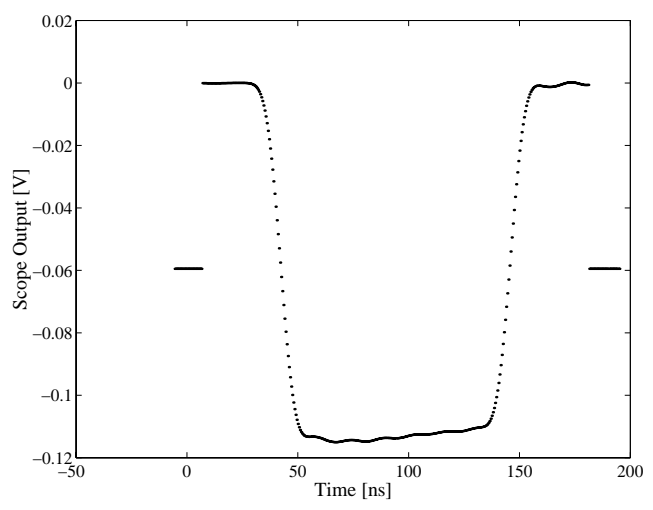

Figure 9: Current Pulse near Beam Edge.

Measurements have also been made under various other conditions (e.g. different accelerating voltage, greater pulse length) but due to space limitations the results of these will not be presented here.

\section{SUMMARY AND FUTURE WORK}

We have been able to develope an experimental method whereby we can temporal, and semi-spatial, resolution of the longitudinal energy distribution in a space-charge dominated electron beam. This, along with other diagnostics in the UMER experiment, give us a means of better understanding the dynamics of space charge dominated charged

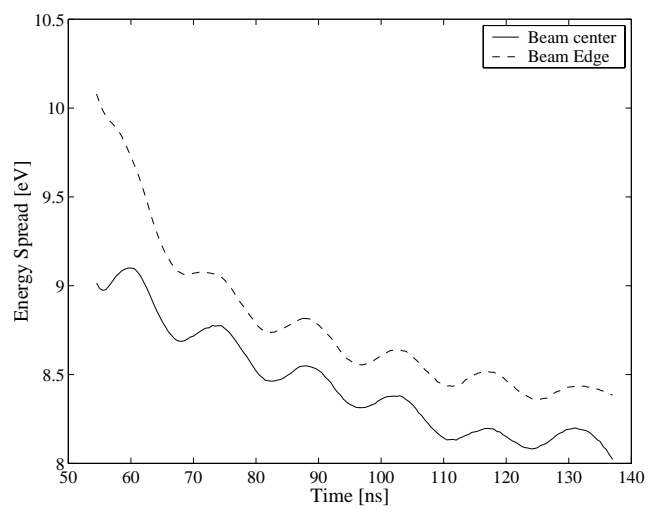

Figure 10: Temporal Variation of Energy Spread.

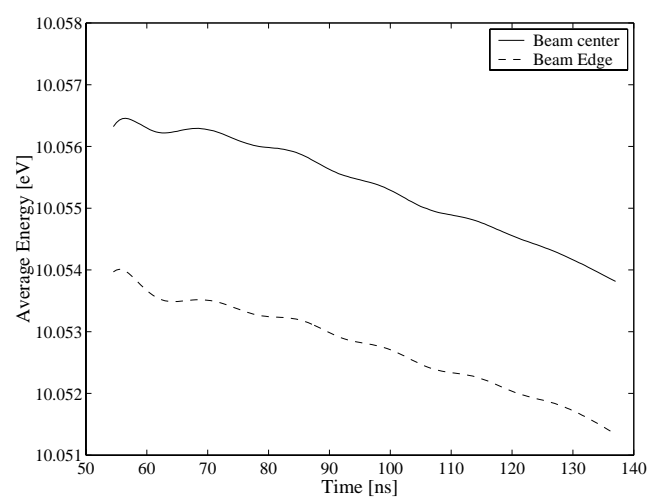

Figure 11: Temporal Variation of Average Energy.

particle beams (such as those used for heavy ion inertial confinement fusion).

We are currently improving our experimental design to improve the resolution of the energy analyzer for this experiment and another related one on energy spread growth [5]. We will then do further energy spread measurements for the UMER injector as well as measurements during construction of the ring section to examine the effect of bending on the energy spread.

\section{REFERENCES}

[1] http://www.ireap.umd.edu/umer

[2] P.G. O'Shea, "The University of Maryland Electron Ring (UMER)", these proceedings.

[3] Yun Zhuo, Ph.D. Dissertation, Department of Electrical Engineering, University of Maryland, 2000.

[4] S. Bernal, R.A. Kishek, M. Reiser, and I. Haber, "Observations and Simulations of Transverse Density Waves in a Collimated Space-Charge Dominated Beam", Phys. Rev. Lett., 82, 4002 (1999).

[5] Y.P. Cui, et al., "Design Studies for an Experiment to Measure Energy Spread Evolution through a Solenoidal Focusing System", these proceedings. 